\title{
NDM: 1-Bit Delta-Sigma Converter with Non-Linear Loop
}

\author{
Miguel A. Lagunas ${ }^{1,2}$, Ana Perez-Neira ${ }^{1,2,}{ }^{*}$, and José Rubio ${ }^{1}$ \\ ${ }^{1}$ Centre Tecnològic de Telecomunicacions de Catalunya (CTTC/CERCA), 08860 Castelldefels, Spain \\ ${ }^{2}$ Universitat Politècnica de Catalunya (UPC), 08034 Barcelona, Spain
}

\begin{abstract}
In this paper we propose to introduce a new processing scheme in the basic loop of a Delta Sigma $(\Delta \Sigma)$ analog-to-digital converter. This processing confers extra gains of the converter over both the quantization error and the channel noise. This is an advance with respect to all cases found in the literature, where the desired signal is not protected against channel noise. Also, the proposed processing is simple and contrasts with the existing architectures, which produce better quality at the expense of sensitivity to implementation imperfections due to the presence of multiples loops in the corresponding architecture. Furthermore, the in-phase/quadrature components structure of a band pass signal has not been used to improve the performance of $\Delta \Sigma$ converters.
\end{abstract}

\section{State of the art}

The $\Delta \Sigma$ converter, sometimes described as 1-bit analogto-digital converter and fully implemented in the audio industry, achieves with a single bit the same quality as a traditional converter of 16 bits. The impact of the $\Delta \Sigma$ technology is tremendous and the number of technical papers on the topic is large. A good paper, widely referenced, with the principles of $\Delta \Sigma$ as well as improvements over the original idea is [1]. The basic loop of a $\Delta \Sigma$ converter is composed of an integrator followed by a two levels quantizer (amplitudes +/- A or "mid-tread") or three levels quantizer (amplitudes $\{0,+\mathrm{A},-\mathrm{A}\}$ named "mid-raiser"). The output signal is feedback to the input, such that the difference $(\Delta$-delta) of the original signal plus the feedback output forms the input to the integrator ( $\Sigma$-sigma). This basic loop implements both oversampling and noise shaping principles in order to produce a discrete version of the original signal. The original signal is recovered by lowpass filtering the discrete version at the output of the $\Delta \Sigma$ converter.

In order to further increase the quality of the basic $\Delta \Sigma$ converter, additional loops can be imbricated in the original scheme. This is referred to as high order $\Delta \Sigma$ converters, where the order reflects the number of feedback loops used or number of "delta" blocks forming the converter. The $\Delta \Sigma$ described in the previous paragraph is denoted as first order $\Delta \Sigma$ converter. High order $\Delta \Sigma$ modulators can also be achieved by cascading first order modulators [2]. Other techniques like time interleaved $\Delta \Sigma$ converters increase also the quality of the first order $\Delta \Sigma$ by setting several order one $\Delta \Sigma$ converters in parallel. In general, all the architectures reported with order greater than one produce better quality at the expense of sensitivity to implementation imperfections due to the presence of multiples loops in the corresponding architecture. Also, non-linear processing, in general frequency (FM) or phase modulators (PM), has been reported implemented as a pre-processing cascaded before the $\Delta \Sigma$ converter. Examples of these converters using FM modulator or Hadamard modulators, can be found in [3-5] and references herein. None of the existing modification alters the basic loop of the $\Delta \Sigma$ converter.

This paper describes a new processing that allows for gains from one bit to five bits, i.e. $15-30 \mathrm{~dB}$ of Signal to Quantization Noise Ratio $\left(\mathrm{SNR}_{\mathrm{Q}}\right)$ over the gains obtained from a first order, let us say, traditional $\Delta \Sigma$ or converter, yet preserving the oversampling and noise shaping gains of these converters. Aside from achieving a technique of low complexity for analog-to-digital conversion, the proposed technique provides significant reduction on both the quantization noise and the channel noise. This additional protection to the channel noise is an advance with respect to all cases found in the literature. The main feature of the proposed technique is that the signal after the delta operation, followed by the sigma operation, and before entering into the quantizer, is modulated in phase or in frequency. Furthermore, the proposed architecture introduces also the inphase/quadrature structure of a band-pass signal, which, to the authors' knowledge, has not been used yet to improve the performance of $\Delta \Sigma$ converters. The proposed technique is called: Non-linear delta sigma modulation or NDM. Furthermore, NDM-PM will denote a phase modulation transformation and NDM-FM a frequency modulation transformation.

\section{Proposed NDM}

Let us consider a generic low-pass and band-limited real signal $x(t)$, with bandwidth B Hz. Either this signal or its

\footnotetext{
* Corresponding author: aperez@cttc.es
} 
sample and hold version, $x(n)$, are considered as the data input to the proposed converter. In the latter case, the sampling rate is $\mathrm{B}_{\mathrm{T}}$ samples/sec, being the quotient $\mathrm{B}_{\mathrm{T}} / 2 \mathrm{~B}$ the so-called oversampling ratio. For the sake of simplicity we consider that the sample period is normalized to one. The input signal has a dynamic range equal to $+/-1 \mathrm{mV}$.

\section{$1.2 \mathrm{I}-\mathrm{Q}$ modulator}

Figure 1 shows the proposed architecture. Let us first describe the upper part of the figure, where the NDM loop is depicted. The delta operation produces the signal $u(n)$ as the difference between the input signal $x(n)$ and the previous sample of $z(n)$ :

$$
u(n)=x(n)-z(n-1)
$$
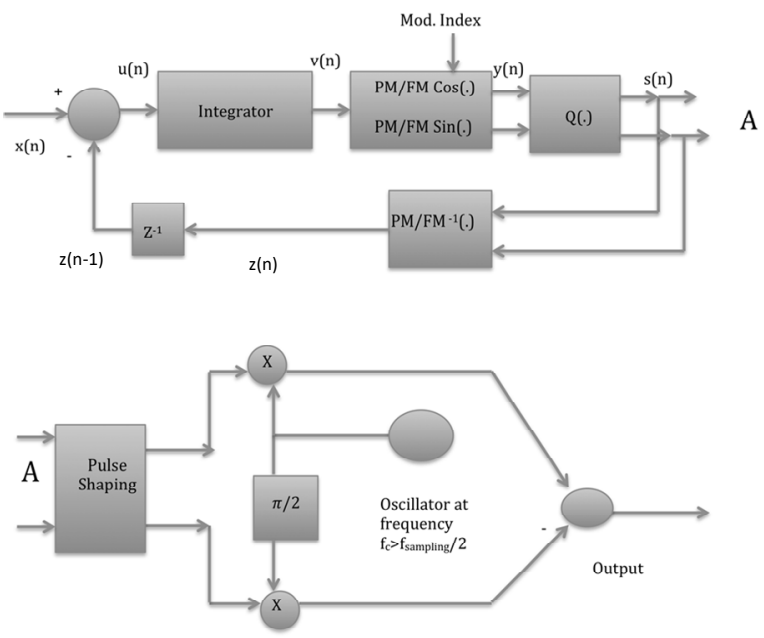

Fig. 1. Proposed 1-bit delta-sigma converter with non-linear loop.

The sigma operation is implemented by an integrator that produces $v(n)$ as:

$$
v(n)=v(n-1)+u(n)
$$

that, by substituting $\mathrm{u}(\mathrm{n})$ as formulated in (1), results in

$$
v(n)-v(n-1)=x(n)-z(n-1)
$$

For PM modulation (NDM-PM), the modulator forms the in-phase and quadrature components as: $\cos \left(\phi_{d} v(n)\right)$ and $\sin \left(\phi_{d} v(n)\right)$ respectively, where $\phi_{d}$ is the phase modulation index. For FM modulation (NDM$\mathrm{FM}), v(n)$ is integrated and the resulting signal $v \mathrm{fm}(n)$

$$
v f m(n)=v f m(n-1)+v(n)
$$

modulates the in-phase and quadrature components as: $\cos \left(2 \pi f_{d} v f m(n)\right)$ and $\sin \left(2 \pi f_{d} v f m(n)\right)$ respectively, where $f_{d}$ is the modulation index.

The pair of in-phase and quadrature components can be represented as the real and imaginary parts of the following complex signal $y(n)$ for PM and FM, which are formulated in equation (5) and (6), respectively

$$
y(n)=\operatorname{PM}[v(n)]=\exp \left(j \phi_{d} v(n)\right)
$$

$$
y(n)=\operatorname{FM}[v(n)]=\exp \left(2 \pi f_{d} v f m(n)\right)
$$

The exponential function converts a sum in a product and a subtraction in a quotient, both for PM and FM. Without loss of generality the NDM-PM is used from now on. The expression of $v(n)$ in the NDM-PM converter can be re-formulated as (7) and (8):

$$
\begin{gathered}
\operatorname{PM}[v(n)-v(n-1)]=\operatorname{PM}[x(n)-z(n-1)] \\
\operatorname{PM}[v(n)] / \operatorname{PM}[v(n-1)]=\operatorname{PM}[x(n)] / \operatorname{PM}[z(n-1)]
\end{gathered}
$$

Taking into account the expression of $y(n)$ in equation (5), the following result is obtained:

$$
\mathrm{y}(n) / \mathrm{y}(n-1)=\operatorname{PM}[x(n)] / \operatorname{PM}[z(n-1)]
$$

After the quantizer, if it is modeled as source of additive noise with complex value $w(n)$, the resulting complex signal $s(n)$ is:

$$
s(n)=y(n)+w(n)
$$

Note that the previous equations imply that the quantizer is linearized. In other words, it is assumed that the quantization noise is independent of the input value. This is not formally correct for low number of quantization levels. Nevertheless, linearizing the quantizer provides light into the resulting performance of the converter even for low number of levels, when the independence claimed is not correct.

Combining equations (9) and (10), the output of the NDM-PM is obtained as a function of the input $x(n)$

$$
\operatorname{PM}[x(n)] / \operatorname{PM}[z(n-1)]=\mathrm{s}(n)-w(n) / \mathrm{s}(n-1)-w(n-1)
$$

In addition, since $z(n)$ is the PM inverse of $s(n)$, we arrive to the final expression that follows:

$$
\mathrm{s}(n)-w(n)=(\mathrm{s}(n-1)-w(n-1)) \operatorname{PM}[x(n)] / s(n-1)
$$

or

$\mathrm{s}(n)=\mathrm{PM}[x(n)])+w(n)-w(n-1) \operatorname{PM}[x(n)] / s(n-1)$

Note that in figure 1 the two branches denoted under $s(n)$ correspond to the in-phase and quadrature components. Aslo, it can be shown that this expression remains the same for the FM modulator just changing the function PM[ . ] by FM[ . ].

Thus $s(n)$ is a discrete version of a phase modulation or frequency modulation of the original signal plus a noise factor. The noise suffers the desired noise shaping, (i.e. loaded to high frequency due to the minus sign affecting the one sample delayed version of it). The difference in the shaping is that this factor is one for $\Delta \Sigma$ and in the proposed scheme the delayed noise sample is multiplied by the quotient between the desired signal (i.e. the PM or FM version of the input $x(n)$ ), divided by the previous output $s(n-1)$. Clearly under low noise regime and high oversampling factor $\left(\mathrm{SNR}_{\mathrm{Q}}\right.$ greater than $30 \mathrm{~dB}$ and $r>5$, where $r=\log _{2}(\mathrm{BT} / 2 \mathrm{~B})$ ), shaping 
becomes almost identical to the obtained on the traditional $\Delta \Sigma$ converter.

It is worth to mention that the phase modulation step, i.e. passing from the output of the integrator to the final phase of the carrier output, can be done directly from the use of a look-up table which resumes the PM modulation and the quantizer. This reduces greatly the complexity of the PM modulator whenever the number of levels is small (i.e. two or three levels quantizers). This applies also for the demodulation embedded in the lower branch. In fact the look-up table passes directly from the input of the modulator to the reconstructed phase values at the lower branch.

Under this model the performance of the NDM versus the conventional $\Delta \Sigma$ improves due to the modulation. For PM the improvement is approximately $20 \log 10\left(\phi_{d}\right) \mathrm{dB}$, which will be close to $10 \mathrm{~dB}$ (i.e. 9.94 $\mathrm{dB}$ ) for $\phi_{d}$ equal to its maximum value of $\pi$, and for FM will be approximately $10 \log 10\left(3\left(f_{d} / \mathrm{B}\right) 2\right)$, where $\mathrm{B}$ is the bandwidth of the original signal. For instance, if $f_{d}=0.2$ and $\mathrm{B}=0.0039$, the improvement versus the conventional $\Delta \Sigma$ converter is $34 \mathrm{~dB}$.

Due to the complex nature of $s(n)$, there are two quantizers acting in parallel, therefore, for a given number of bits for each quantizer the number of used levels is twice that of the traditional $\Delta \Sigma$ converter. For a number of bits in the quantizers greater than 2 (i.e. more than 3 levels per quantizer) this implies an extra gain of $6 \mathrm{~dB}$ due to the fact that the number of levels used in NDM is twice the number of levels used in $\Delta \Sigma$. For the case of 1 bit, the quantization noises and original signal are correlated and no gain is expected due to the use of two quantizers in parallel. As it has already been commented, it is the first time that the in-phase and quadrature structure is used to improve the performance of $\Delta \Sigma$ converters.

\subsection{FM and the threshold effect}

The threshold effect of FM for low SNR is well described in the literature, for example [6]. Basically, the effect is motivated by the use of high modulation index $f_{d}$, in consequence by the large detection bandwidths. This threshold effect appears as peaks or glitches when detecting the instantaneous frequency that degrade the recovered SNR. This causes, for example, that using a mid-raiser (2 levels) quantizer for 1-bit NDM-FM converter presents lower SNR than expected due to the threshold effect. For NDM-PM there is not threshold effect for the oversampling ratio used in the figures, independently of the number of levels of the quantizer.

To overcome the threshold effect, as it is the case of NDM-FM 1-bit mid-riser for the signal scenario selected, it is necessary a phase loop which reduces the processing bandwidth and restores the SNR of the converter.

\section{$1.3 \mathrm{I}-\mathrm{Q}$ modulator}

Figure 1 shows the band-pass modulator as part of the proposed converter, since it is required due to the intrinsic band-pass nature of the PM and FM modulations. The low-pass equivalent signal $s(n)$, at the top of the figure, is modulated, after pulse shaping, by a carrier frequency $f_{c}$ that has to be above half of the sampling frequency. The line signal or output is

Output $(t)=s_{i}(n) \cos \left(2 \pi f_{c} t\right)-s_{q}(n) \sin \left(2 \pi f_{c} t\right)$

In the case of the NDM-PM converter, and for a two levels quantizer, the output is in fact a 4 PSK (Phase Shift Keying) modulation with phases equal to $\pm \pi / 4, \pm 3 \pi / 4$. For the NDM-FM case, the line signal is like a DPSK (differential PSK) over the previous four-point constellation of phases. Note that for the mid-tread (3 levels) quantizer the constellations of phases pass to be $\pm \pi / 8, \pm 3 \pi / 8, \pm 5 \pi / 8,7 \pi / 8$. In other words, it is a 8 PSK (constellation of 8 phases) signal. In consequence, the proposed scheme can be encompassed as a device converting an analog signal directly to a digital bandpass PSK modulation.

Phase unwrapping can be included at the converter reducing the recovery of the original signal to a phase/frequency detector and low pass filtering, i.e. no DAC converter is needed as it is the case in traditional $\Delta \Sigma$ modulators.

\section{Simulations}

Let us consider a signal of bandwidth $\mathrm{B}=0.039$ and carrier frequency of the NDM equal to $f_{c}=0.002$, both normalized to the sampling frequency. If NDM-PM (mid-riser) is used $\phi_{d}=\pi$ and in NDM-FM (mid-tread) $f_{c}=0.2$. The benchmark is a conventional $\Delta \Sigma$ converter (mid-riser), whose $\mathrm{SNR}_{\mathrm{Q}}$ coincides with the formula given in [7] and is given by

$S N R_{Q}(d B)=3.01 n(2 L+1)-9.36 L-2.76$.

For order $L=1$ (i.e. 1 bit conversion) and oversampling factor equal to

$$
n=\log _{2}(1 / 2 \times 0.0039)=7
$$

a benchmark of $S N R_{Q}=51 \mathrm{~dB}$ is obtained. Table 1 compares this benchmark with the proposed NDM of 1 bit. Note that the high obtained gains, which are very close to the measured gains.

Table 1. Comparison of the proposed NDM 1-bit converter for both NDM-PM $\left(\phi_{d}=\pi\right)$ and NDM-FM $\left(f_{c}=0.2\right)$. The benchmark is $\Delta \Sigma$ converter of 1 bit.

\begin{tabular}{|c|c|c|c|}
\hline & $\begin{array}{c}\Delta \Sigma \\
\text { converter } \\
\text { of 1 bit }\end{array}$ & $\begin{array}{c}\text { NDM-PM } \\
\text { of 1 bit }\end{array}$ & $\begin{array}{c}\text { NDM-FM of } \\
1 \text { bit }\end{array}$ \\
\hline Measured & $51 \mathrm{~dB}$ & $77.12 \mathrm{~dB}$ & $107.52 \mathrm{~dB}$ \\
\hline $\begin{array}{c}\text { Obtained } \\
\text { Gain }\end{array}$ & - & $26.12 \mathrm{~dB}$ & $56.52 \mathrm{~dB}$ \\
\hline $\begin{array}{c}\text { Theoretical } \\
\text { Gain }\end{array}$ & - & $28.06 \mathrm{~dB}$ & $58.12 \mathrm{~dB}$ \\
\hline
\end{tabular}


Next, for the sake of illustration, figure 2 shows the power spectrum density of the resulting signal $s(n)$ of the NDM converter (NDM-PM converter) in a particular case. The power spectrum density is computed from the average of periodograms of records of 256 samples of the output signal $s(n)$, for the quadrature component only (the power spectrum density for the phase component will be the same). The record length is 105 samples. The input signal is noise of uniform distribution filtered at $\mathrm{B}=0.0039$. The dynamic range of the signal is $+/-1 \mathrm{mV}$. The parameters used in the converter have been: A PM modulator with modulation index $\phi_{d}=\pi$ (maximum phase deviation), carrier amplitude 1 volt, sampling rate normalized to one, 1 bit mid-riser quantizer of 2 levels for both in-phase and quadrature components. The decision levels of the quantizer were $+/-0.5 \mathrm{mV}$. The input signal was white noise with a FIR of 512 coefficients with a bandwidth $B=0.0039$. The plot shows the averaged periodogram in $\mathrm{dB}$ versus the frequency between zero and the sampling frequency equal to 1 (symmetric with respect half the sampling frequency) The resolution of the frequency plot is 9.76 10-5.

In the bottom part of figure 2, the corresponding power spectrum for $\Delta \Sigma$ converter with 1-bit mid-riser 2 levels quantizer is shown.
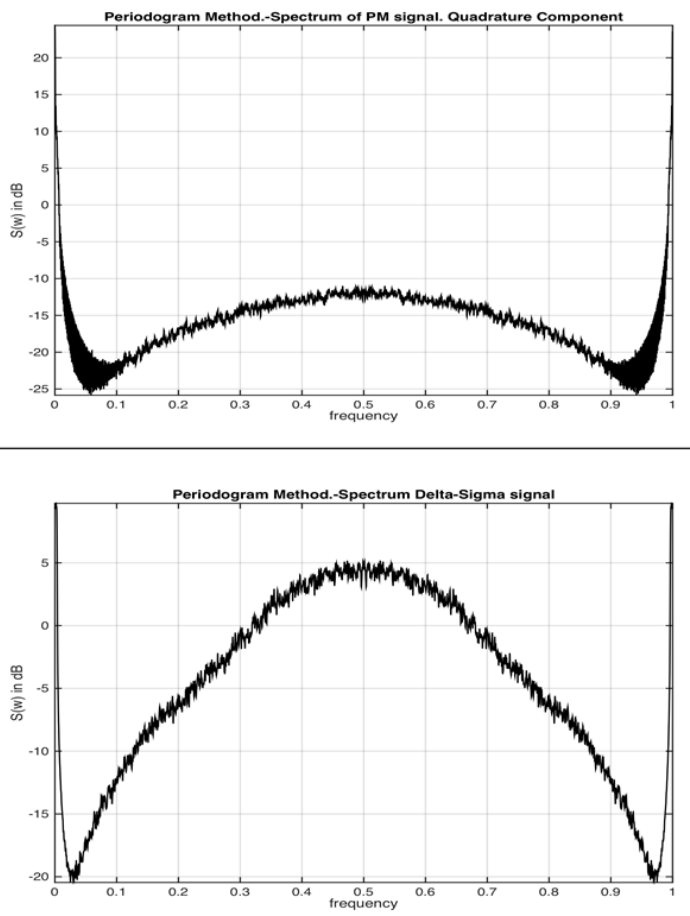

Fig. 2. Power spectrum density of the resulting signal $s(n)$ of the NDM-PM 1-bit converter (upper plot) and of the $\Delta \Sigma$ converter 1-bit mid-riser 2 levels.

Note that the noise shaping is well present in both converters. This effect is masked partially for NDM-PM due to the high spectral dynamic range of the PM signal. Note that the power of output signal is the same in $\Delta \Sigma$ and NDM converters, in consequence the area of both spectral densities are the same. Of course, the selected carrier frequency does not affect the final $\mathrm{SNR}_{\mathrm{Q}}$ quality of the converter since, at the receiver site, the PM detector extracts the unwrapped carrier phase. In the case of NDM-FM we note that the same noise shaping is preserved, for the sake of brevity we omit the plot. In, figure 3 , the quantized signals are plot when $x(t)$ is a sinusoidal waveform.
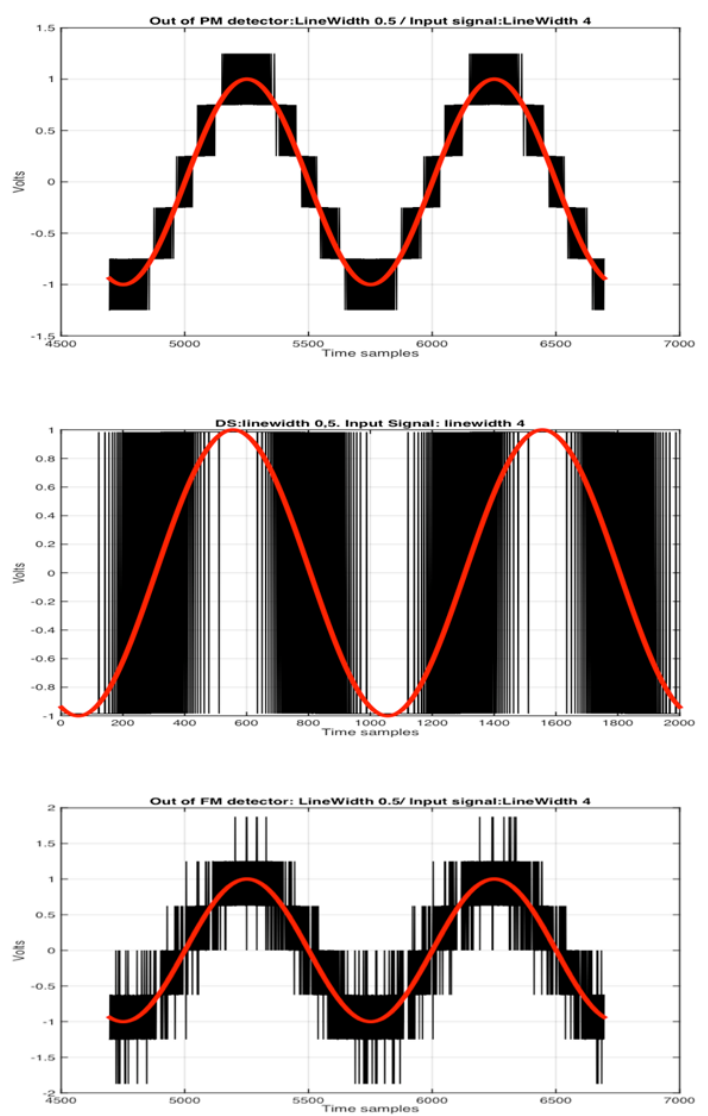

Fig. 3. Quantized sinusoidal waveform with NDM-PM 1-bit (upper plot), $\Delta \Sigma$ 1-bit converter (middle plot), and NDMFM 1-bit (lower plot).

High order NDM can be implemented by the addition of extra delta-sigma loops. Table 2 shows the gains with respect to the equivalent $\Delta \Sigma$ converter and the same settings as in the other simulations, but changing the order and number of bits as it is indicated.

Table 2. Converters of order $\mathrm{L}=2$. Comparison of the proposed NDM for both NDM-PM $\left(\phi_{d}=\pi\right)$ with the $\Delta \Sigma$ converter.

\begin{tabular}{|c|c|c|}
\hline $\begin{array}{c}\text { Number } \\
\text { of bits }\end{array}$ & $\begin{array}{c}\text { SNR } \Delta \Sigma \\
(\mathrm{L}=2)\end{array}$ & $\begin{array}{c}\text { SNR NDM- } \\
\text { PM }(\mathrm{L}=2)\end{array}$ \\
\hline 1 & $84.23 \mathrm{~dB}$ & $115.16 \mathrm{~dB}$ \\
\hline 2 & $92.58 \mathrm{~dB}$ & $117.42 \mathrm{~dB}$ \\
\hline 3 & $96.65 \mathrm{~dB}$ & $124.16 \mathrm{~dB}$ \\
\hline 4 & $98.03 \mathrm{~dB}$ & $129.93 \mathrm{~dB}$ \\
\hline
\end{tabular}


It is worth mentioning that NDM-FM performs similar to NDM-PM $\left(\phi_{d}=\pi\right)$ when the FM modulator uses an index equal to 0.025 . Note that NDM-FM entails the use of two integrators, i.e. one of the loop and another in the modulator, as well as NDM-PM of order two. Amazingly NDM-PM of order two provides better $\mathrm{SNR}_{\mathrm{Q}}$ than NDM-FM of order one. It is important to mention that NDM-PM entails a narrow band modulation meanwhile NDM-FM is wideband without taking full advantage of the noise shaping. In addition, note that the receiver for FM is simpler than for PM, furthermore the receiver for FM can be fully analog and un-coherent.

\section{Conclusions}

This paper proposes a new processing scheme in the basic loop of a Delta Sigma $(\Delta \Sigma)$ analog-to-digital converter. This processing confers extra gains of the converter over both the quantization error and the channel noise. This latter aspect is completely new in the existing literature. The proposed processing is simple and, furthermore, it incorporates the in-phase/quadrature components structure of a band pass signal, which is the first time that it is used to improve the performance of $\Delta \Sigma$ converters. The presented work has been filed as a patent in [8]. Some of the advantages of the presented converter proposed are, for example:

- High dynamics (50 -100 dB) obtained by low bit converters are currently fully installed on audio signal sensing and distribution. In addition to the high dynamics, the major innovation of this work relies on the ability to convert directly a time continuous signal, or a sample/hold version of it, in a constant envelope digital modulated waveform. The resulting waveform is a constant envelope carrier modulated either in phase or frequency (PSK or FSK). This implies that the converter implements a source coding or representation in terms of a finite set of phases in the i/q (in-phase and quadrature) components of a pre-selected carrier frequency, i.e. channel coding. This enables to interchange between communications nodes high dynamic range signals and, at the same time, providing protection of the coded signal against radio-channel noise of the coded signal.

- The impact, especially in Internet of Things (IoT) networks, is very high since the sensing part is almost independent of the corresponding RF part with the corresponding limitations in performance. In addition, the invention represents a way out to the, let us say, traditional, low dynamic and low bandwidth that ambient sensors use to handle. Therefore, the proposed converter represents an innovation impacting the wireless and wired sensor production from distributed acoustic processing down to ambient sensing.

- The NDM-FM converter can produce narrowband Frequency Modulation (small fd) enabling the converter to work with a wide range of transmission bandwidths for the desired signal.

- The angle modulations embedded in NDM converters protects the NDM converted signal from channel noise, whenever the phase/frequency detector stays above the threshold effect.

- The increase in the power of the line signal, PM/FM modulated, produces greater protection to channel noise than linear modulations.

- The in-phase and quadrature components in the proposed NDM converter are quantized independently allowing either the arrangement in a single carrier in wireless transmission or the time multiplexing of the two streams, yet preserving its superiority with respect to traditional $\Delta \Sigma$ converter (double oversampling factor).

- The NDM converter can be easily extended to high order modulators NDM-PM and NDM/FM.

- NDM-PM modulators of order $L+1$ are better than NDM-FM of order $L$, with slightly lower complexity than NDM-FM. At the same time the spectral bandwidth of NDM-PM desired signal shows better spectrum confinement for the desired that NDM-FM.

- The NDM converter implements a joint source channel coding that converts a baseband analog signal in a high rate digital phase modulation (QPSK or 8PSK for 1 bit per quantizer).

This work has received funding from the Spanish Government under project TERESA TEC2017-90093-C3-1-R, and from the Catalan Government (2017 SGR 891 and 2017 SGR 1479.

\section{References}

1. P. M. Aziz, H.V. Sorensen, J.Van der Spiegel. "An overview of Sigma-Delta Converters: How 1-bit ADC achieves more than 16-bit resolution," IEEE Signal Processing Magazine, 13, Issue 1, pp. 61-84, September (1996).

2. I. Galton and H. T. Jensen, "Delta-Sigma modulator based A/D conversion without oversampling," IEEE Transactions on Circuits and Systems II: Analog and Digital Signal Processing, vol. 42, no. 12, pp. 773-784, December (1995).

3. M. Pedersen. "Circuit for direct digital delta-sigma conversion of variable electrical capacitance," US Patent. US 69575258 B2, Dec. (2005).

4. M Hovin, A. Olsen, T. Sverre Lande, C. Toumazou. "Delta-Sigma Modulators using FrequencyModulated Intermediate Values," IEEE Journal of Solid State Circuits, 32, No. 1, Jan. (1997).

5. M Hovin, A. Olsen, T. Sverre Lande, C. Toumazou. "Delta-Sigma Modulators using FrequencyModulated Intermediate Values," IEEE Journal of Solid State Circuits, 32, No. 1, Jan. (1997).

6. B. Carlson, "Communication Systems: An introduction to Signals and noise in Electrical Communication, McGraw-Hill (1986).

7. D. Van Ess, "Signal from Noise: Calculating DeltaSigma SNRs," EN-Genius Network. www.engenius.net.

8. Patent filing $\mathrm{N}^{\mathrm{o}} \mathrm{PCT} / \mathrm{EP} 2016 / 068402$. 